\title{
Assessing Segregation Effects on Multiferroic Properties of Antiferromagnetic- Weak Ferromagnetic Coupled Systems by Analytical HRTEM
}

\author{
J. M. Vieira ${ }^{1}$, A. Baghizadeh ${ }^{1}$, J. S. Amaral ${ }^{2}$, J. N. Gonçalves ${ }^{2}$, B. A. Almeida ${ }^{1}$, M-G. Willinger ${ }^{3}$ and V. S. Amaral ${ }^{2}$ \\ ${ }^{1 .}$ Department of Materials and Ceramic Engineering \& CICECO, University of Aveiro, 3810-193 Aveiro, \\ Portugal. \\ 2. Physics Department \& CICECO, University of Aveiro, 3810-193 Aveiro, Portugal. \\ ${ }^{3 .}$ Fritz Haber Institute of the Max Planck Society, Department of Inorganic Chemistry, Faradayweg 4-6, 14195 \\ Berlin, Germany.
}

The interplay of ferroic domain walls with crystalline structures and lattice planar defects becomes rather elaborated in multiferroic materials or in magnetic bilayers and multilayers where magnetic moments of antiferromagnetic and ferromagnetic lattices are coupled at the bilayer interface [1-5]. Single atomic layer and double layers make the antiphase ferroelectric domain walls organized in six-fold vortices in multiferroic materials of the class of the hexagonal rare-earth manganites $\mathrm{RMnO}_{3}[4,6]$. Below $\mathrm{T}_{\mathrm{N}}$, the Néel temperature, antiferromagnetic domain walls are coupled to the same antiphase domain walls $[3,7,8]$. Magnetoelectric coupling exposes the multiferroic nature of these materials [3,4,7]. In addition, the same hexagonal $\mathrm{RMnO}_{3}$ materials often display weak-ferromagnetism of which the origin is poorly understood $[1,8,9]$.

The resolution of aberration-corrected electron microscopy, mostly the high angle annular dark field scanning transmission microscopy ADF-STEM permitted direct observation of ferroelectric switching in hexagonal $\mathrm{RMnO}_{3}$ and corroborated the topology of multiferroic vortex domains established from more conventional TEM and sensor force microscopy $[1,3,4,6,7,10,11]$. It also exposed the role of partial-edge dislocations and planar defects in breaking the ideal topology of the multiferroic vortices in the same materials [11]. Even when $\mathrm{RMnO}_{3}$ materials are prepared as single crystals by floating zone methods differences between composition of congruent melting of the hexagonal manganites and the stoichiometry of the nominally pure phase contribute to segregation of the constituents of the crystal. Relevance of segregation effects rises from single crystals to thin films and it is definitively higher in bulk polycrystalline materials. Composition of the hexagonal phase of $\mathrm{RMnO}_{3}(\mathrm{R}=\mathrm{Lu})$ ceramics was intentionally deviated from stoichiometry in a study of the magnetic and dielectric behavior of the multiferroic material. Samples were prepared by high temperature synthesis by reaction of the $\mathrm{Lu}_{2} \mathrm{O}_{3}$ and $\mathrm{Mn}_{3} \mathrm{O}_{4}$ compounds. Irreversibility of field cooled (FC) and zero field cooled (ZFC) magnetization below $\mathrm{T}_{\mathrm{N}}$ is strongly correlated to shifts of stoichiometry. A weak ferromagnetic component of magnetization is detected overlapping with antiferromagnetic ordering. Atomic resolution imaging of thin sections of the crystalline grains of $\mathrm{RMnO}_{3}(\mathrm{R}$ $=\mathrm{Lu})$ ceramics by HR-TEM and BF-STEM showed a high density of distortion in the crystalline lattice with the form of a texture of nanodomains with the scale of few dozen nanometers. By increasing the time of high temperature annealing from one day to ten days at $1300{ }^{\circ} \mathrm{C}$ the texture observed inside the crystalline grains could not be eliminated. In spite of definitively poorer resolution of non-corrected TEM, energy filtered TEM imaging along [110] zone axis of $\mathrm{LuMnO}_{3}$ allowed observation of phase shifts that identify anti-phase domain walls (DW) of ferroelectric domains, Figure 1. The intragrain distortion of the crystalline lattice is also represented by twinning, antiphase boundaries, dislocations and atomic clusters. Electron energy loss spectrometry EELS showed that oxidation state of the $\mathrm{Mn}$ ion in self-doped $\mathrm{LuMn}_{\mathrm{x}} \mathrm{O}_{3 \pm \delta}$ lattice remains close to $\mathrm{Mn}^{3+}$. Elemental mapping by EDS in STEM exhibited chemical inhomogeneity inside the crystalline grains at different scales: as nanosized Mn rich clusters, Figure1, and as gradients of the $\mathrm{Mn} / \mathrm{Lu}$ ratio of wider scales. As for the partial edge dislocations, the strain field of nanoclusters (Figure 1) also blocks ferroelectric anti-phase domain walls (DW) and breaks the topology of the multiferroic vortex [11]. 
The intensity of weak ferromagnetism in $\mathrm{LuMn}_{\mathrm{x}} \mathrm{O}_{3 \pm \delta}$ ceramics is enhanced on the Mn-rich side of the solid solution. Segments of ferroelectric anti-phase domain walls may become electrically charged [10]. By interacting with effectively charged point defects they may react to the shifts of stoichiometry. Weak magnetization created by domain wall magnetoelectric effect would change by the effect of segregation of charged point defects to the domain walls [8]. Single atomic column (SAC) EELS in STEM was used to determine atom substitution at domain walls [12]. The feasibility of testing changes of ferroelectric domain wall effective charging by (SAC) EELS has to be demonstrated yet. Due to chemical heterogeneity an extrinsic cause may explain the weak ferromagnetism below $43 \mathrm{~K}$. It may simply originate from $\mathrm{Mn}_{3} \mathrm{O}_{4}$ impurity retained by incomplete reaction or as intergrowths with $\mathrm{LuMnO}_{3}$. Electron back-scatter diffraction (EBSD) in SEM better than EDS guided the search for scarce $\mathrm{Mn}_{3} \mathrm{O}_{4}$ inclusions in the TEM thin sections. As the core-shell nanoparticles of $\mathrm{MnO} / \mathrm{Mn}_{3} \mathrm{O}_{4}$ system, exchange coupling through the interface of ferrimagnetic $\mathrm{Mn}_{3} \mathrm{O}_{4}$ and antiferromagnetic $\mathrm{LuMnO}_{3}$ main phase may be correlated to the observed exchange bias of magnetism [5,9]. Elemental profiling by EDS in STEM of the area of the $\mathrm{Mn}_{3} \mathrm{O}_{4} / \mathrm{LuMnO}_{3}$ interface showed gradients of element concentration that corroborates the presence of active transport by interdiffusion in both phases across a rather regular solid-solid interface. The role of interfaces in the nanostructure in the weak ferromagnetism above $43 \mathrm{~K}$ is further considered [13].

References:

[1] T Kastufuji et al., Phys. Rev. B 64 (2001) 104419 (6pp)

[2] X W Wu and C L Chien, Phys. Rev. Lett. 81 (1998) p. 2795.

[3] M Fiebig et al., Nature 419, (2002) 08E302 p. 818.

[4] T Choi et al., Nat Mater 9 (2010) p. 253.

[5] A E Berkowitz et al., J. Phys. D: Appl. Phys. 41 (2008) 134007 (5pp)

[6] Q Zhang et al., Sci. Rep. 3 (2013) 2741 (5pp)

[7] Y Geng et al., Nano Lett. 12 (2012) p. 6055.

[8] H Das et al., Nat. Comm. 5 (2014) 2998 (25pp)

[9] A A Belik, J. Phys. Soc. Jpn. 83 (2014) 074703 (7pp)

[10] M G Han et al., Adv. Mater. 25 (2013) p. 2415.

[11] Q Zhang et al., App. Phys. Lett. 105 (2014) 012902 (11pp)

[12] S Farokhipoor et al., Nature 515 (2014) p. 379.

[13] Acknowledged support from FCT/MEC project PTDC/FIS/105416/2008 "MULTIFOX", CICECO-Aveiro Institute of Materials (Ref. FCT UID /CTM /50011/2013), (REDE/1509/RME/2005) and fellowships

SFRH/BPD/80663/2011 and SFRH/BPD/63942/2009.

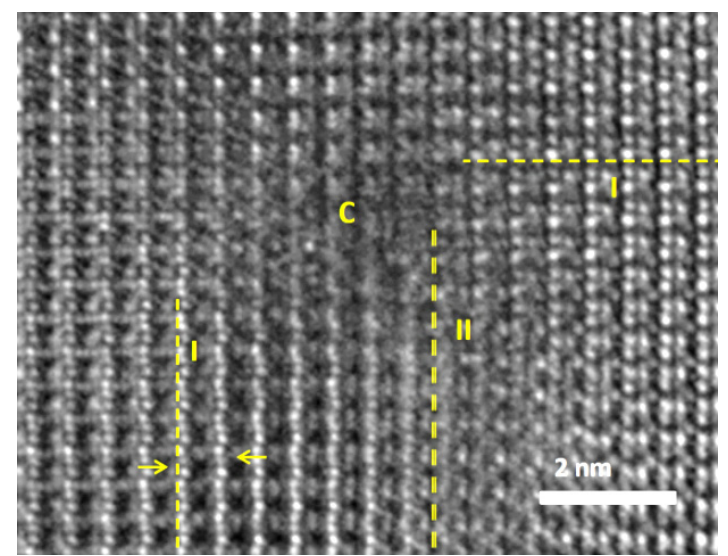

Figure 1. [110] zone axis view of energy filtered TEM of $\mathrm{LuMn}_{0.92} \mathrm{O}_{3 \pm \delta}$ ceramic sample with atomic cluster (C) of increased Mn concentration in relation to average composition of the crystalline lattice, as determined by EDS. The position of three antiphase ferroelectric boundaries of types (I) and (II) are shown by the dashed lines, the types of $\mathrm{APB} / \mathrm{FEB}$ in $\mathrm{h}-\mathrm{RMnO}_{3}$ multiferroics being defined in references $[4,6]$. 